\title{
Face-on SS 433 Stars as a Possible New Type of Extragalactic X-Ray Sources
}

\section{S. Fabrika}

Special Astrophysical Observatory, Nizhnij Arkhyz, Russia

A. Mescheryakov

Sternberg Astronomical Institute, Moscow, Russia

\begin{abstract}
The object SS 433 is a well-known source of relativistic jets, which are formed in supercritical accretion disk. It is very probable that the disk has polar channels and their radiation is collimated (the photocones). A face-on SS433 object can appear as ultra-bright and highly variable X-ray source, $L_{x} \sim 10^{40}-10^{42} \mathrm{erg} / \mathrm{s}$. We discuss the properties of these hypothetical objects and their frequency expected in galaxies. We describe a search for such objects using the ROSAT All Sky Survey and RC3 catalog of galaxies. Among the total 418 positive correlations we find that 142 sources in S and Irr galaxies are unknown as AGNs. Nuclear sources among them still contain many AGNs. Non-nuclear (offset) sources are rather hard, their X-ray luminosities are $10^{39}-10^{41} \mathrm{erg} / \mathrm{s}$. Their observed frequency is about $4--5 \%$ per galaxy, that is in agreement with expected frequency of the face-on SS 433 stars. The only way to recognize such stars is their expected violent variability in $\mathrm{X}$-rays.
\end{abstract}

The well-known galactic binary star SS 433 shows unique relativistic jets moving with a velocity $V_{j}=0.26 c$. The jets are originated in the supercritical accretion disk. The disk is extremely bright, its total luminosity is $L_{b o l} \sim$ $10^{40} \mathrm{erg} / \mathrm{s}$ with maximum of the radiation in UV (Dolan et al. 1997). The jets propagating in inner regions of the disk's wind make channels, which may form a collimated radiation of SS 433. Such a star being orientated "face-on" and observed in photocone will look brighter by brightening factor $B=2 \pi / \Omega_{c}$, where $\Omega_{c}$ is solid angle of the channel. It will be X-ray source registered with a luminosity $B L_{c}$, where the cone luminosity is not easy to estimate. We may expect $L_{c} \sim L_{b o l}$ and accept here $L_{c}=L_{b o l}$.

We search for hypothetical objects, face-on SS 433 stars, which as we expect, have to be ultraliminous $\mathrm{X}$-ray sources. Their expected properties are: i) frequency is $1 / B$ per spiral galaxy like $\mathrm{MW}$; ii) location is in spiral galaxies (arms, star-burst regions), Irr galaxies, nuclear regions of spiral and S0 galaxies; iii) X-ray luminosity is ultra-high, $10^{40} \div 10^{42} \mathrm{erg} / \mathrm{s}$. Spectrum is unknown, probably soft. Very variable, sporadical variability from $1 \mathrm{~min}$ to 1 year, periodical variability with orbital and precession periods (days, months). UV or blue star, very faint in visible, $V \gtrless 21^{m}$ for $D=10 \mathrm{Mpc}$.

We have correlated RC3 catalogue of galaxies (de Vaucouleurs et al. 1991) with ROSAT All Sky Survey Bright Source Catalogue (BSC, Voges et al. 1999) 
and Faint Source Catalogue (FCS, Voges et al. 2000). RC3 contains 23007 galaxies (15415 spirals and 1326 irregulars). The Local Group galaxies were omitted. RASS $(0.1-2.4 \mathrm{kev})$ contains 18811 sources in BSC $(F>0.05 \mathrm{cts} / \mathrm{s})$, 8365 among them are point-like and 91163 point-like sources in FSC.

The correlation box was used as 25 th magnitude isophote of a galaxy + Rerr. A number of contaminations was found making multiple correlations with galaxy coordinates $+\Delta \alpha=1^{h}, 2^{h}$, etc. We have chosen Rerr $=30^{\prime \prime}$ for final correlations. We find 418 positive correlations (308 sources from FSC and 110 sources from BSC), about 90 sources from them are contaminations. After comparing with Veron-Cetty \& Veron (2000) cataloque we have 327 positive correlations of Xray sources (unknown as AGNs) with RC3 galaxies. Considering only spiral and irregular galaxies, we find the final number of X-ray point-like sources is 142 .

A visual inspection of all 142 BSC + FSC sources in spiral and irregular galaxies has been done. These sources have been classified in 4 groups: 1st class of clear offset (non-nuclear) sources, without probable contamination with a star-like object in X-ray error boxes - 37 sources; 2nd class of probable offset, but may be nuclear sources - 43 sources; 3rd class of very probable nuclear sources, unknown AGNs can be present in this class, - 35 sources; 4th class of probable contaminations (star-like objects inside a box or unusually big distance between X-ray source and galaxy).

A study of hardness ratios has shown, that there are many $\mathrm{X}$-ray sources with hard spectra among our best correlations. Frequency of hard sources grows from nuclear to offset sources. We found also that nuclear sources are brighter. They include really unknowm AGNs. We compared X-ray luminosities and numbers of our sources with offset sources and AGNs from Roberts \& Warwick (2000). This is a study of complete list of nearby galaxies with the HRI of ROSAT. We find that a ratio of offset sources to AGN (nuclear) found from their data is 1.3 , and the same ratio 1.5 in our data.

In FSC we expect to detect all bright sources with $L_{x}>10^{40} \mathrm{erg} / \mathrm{s}$ in galaxies closer that $11 \mathrm{Mpc}$. At distances $>10-20 \mathrm{Mpc}$ we have a selection in our data and can not recognize all sources with $L_{x}>10^{40} \mathrm{erg} / \mathrm{s}$. We conclude that the data do not contradict the idea of existence of the "hypothetical" face-on SS $433 \mathrm{X}$-ray sources. The frequency of the best offset sources (the 1-st class) is about $4 \div 5 \%$ per galaxy. They are ultra-bright X-ray sources, $L_{x} \sim$ $10^{40} \div 10^{41} \mathrm{erg} / \mathrm{s}$. The only way to identify such stars is their expected violent variability.

\section{References}

de Vaucouleurs G. et al. 1991, Third Reference Cataloque of Bright Galaxies (New York: Springer)

Dolan, J.P. et al. 1997, A\&A, 327, 648

Roberts, T.R., \& Warwick, R.S. 2000, MNRAS, 315, 98

Veron-Cetty, M.-P., \& Veron, P. 2000, A catalogue of quasars and active nuclei (9th edition), http://www.obs-hp.fr/

Voges, W. et al. 1999, A\&A, 349, 389

Voges, W. et al. 2000, http://wave.xray.mpe.de/rosat/cataloques/rass-fsc. 